\title{
Poincaré gauge symmetries, hamiltonian symmetries and trivial gauge transformations
}

\author{
Rabin Banerjee* and Debraj Roy ${ }^{\dagger}$ \\ S. N. Bose National Centre for Basic Sciences, \\ Block-JD, Sector III, Salt Lake, Kolkata-700098, India.
}

\begin{abstract}
We resolve a problem of finding the Poincaré symmetries from hamiltonian gauge symmetries constructed through a canonical procedure of handling constrained systems. Through the use of Noether identities corresponding to the symmetries, we motivate a procedure of finding the map between the hamiltonian and Poincaré gauge parameters. Using this map, we show that the Poincaré and hamiltonian gauge symmetries are equivalent, modulo trivial gauge transformations.
\end{abstract}

\section{Introduction}

Gauge symmetries in various diffeomorphism invariant theories are a matter of continued interest. Some, among the multitude of models where gauge symmetries have been studied, are ChernSimons gauge theory [1], Einstein-Cartan gravity [2,3], topological gravity with torsion [4,5] and topologically massive gravities [6,7] including Bergshoeff-Holm-Townsend (BHT) or "new massive gravity" [8 10]. By gauge symmetries we mean those transformations of the basic fields of the action, parametrised by arbitrary functions of time, that leave the action invariant under appropriate boundary conditions [11]. The arbitrary functions of time are the gauge parameters.

On the other hand, diffeomorphism invariant theories have the Poincaré symmetries ' $\delta_{P G T}$ ', i.e. local Lorentz rotations and translations, as off-shell symmetries by construction. They are found in the usual manner of gauge theories, through localisation of the Poincaré symmetries [2,12,15] and so their form dosen't depend on the particular diffeomorphism invariant model being considered. Say, for example, let us first consider the Einstein-Cartan action in 3D

$$
S_{1}=\int \mathrm{d}^{3} \mathrm{x} \epsilon^{\mu \nu \rho} b_{\mu}^{i} R_{i \nu \rho}
$$

and then add to it the torsion $T_{i \nu \rho}$ enforced by a parameter $\alpha_{4}$

$$
S_{2}=\int \mathrm{d}^{3} \mathrm{x} \epsilon^{\mu \nu \rho}\left[b^{i}{ }_{\mu} R_{i \nu \rho}+\frac{\alpha_{4}}{2} b^{i}{ }_{\mu} T_{i \nu \rho}\right]
$$

\footnotetext{
*rabin@bose.res.in

${ }^{\dagger}$ debraj@bose.res.in
} 
where $b^{i}{ }_{\mu}$ is the triad and $R_{i \nu \rho}$ is the Riemann tensor. The Poincaré symmetry of the (for example) triad field is the same for both of these actions

$$
\delta_{P G T} b_{\mu}^{i}=-\epsilon_{j k}^{i} b_{\mu}{ }_{\mu} \theta^{k}-\partial_{\mu} \xi^{\rho} b_{\rho}^{i}-\xi^{\rho} \partial_{\rho} b^{i}{ }_{\mu}
$$

and as we can see, it does not involve the coupling constant $\alpha_{4}$. It is off-shell by construction and this can be easily checked explicitly [5]. The gauge parameters here are $\xi^{\rho}$ for translations and $\theta^{i}$ for local Lorentz rotations.

To study the hamiltonian gauge symmetries ' $\delta_{G}$ ', canonical hamiltonian analysis of all the models mentioned above has been carried out extensively, in the literature cited above (also see references therein). The nature of the hamiltonian symmetries depend intimately on the particular model being studied, through the structural nature of the constraints. These hamiltonian symmetries reveal a striking feature, in all of the models. The Poincaré symmetries are not identifiable from the hamiltonian gauge symmetries. For example, for the Einstein-Cartan action with torsion we get

$$
\delta_{G} b^{i}{ }_{\mu}=\nabla_{\mu} \varepsilon^{i}+\alpha_{4} \epsilon_{j k}^{i} b_{\mu}^{j} \varepsilon^{k}+\epsilon^{i}{ }_{j k} b^{j}{ }_{\mu} \tau^{k},
$$

where $\varepsilon^{i}$ and $\tau^{i}$ are the gauge parameters. Note that $\delta_{G}$ explicitly involves the coupling constant $\alpha_{4}$. To compare $\delta_{P G T}$ and $\delta_{G}$ we first have to map the (arbitrary) gauge parameters of the hamiltonian symmetries $\delta_{G}$ to those of the Poincaré symmetries $\delta_{P G T}$. The gauge parameters become different as the Poincaré parameters are dictated by either geometric or group theoretic demands while the hamiltonian parameters depend on the structure of the constraints arising in the theory. The required redefinition is usually done through an ad-hoc, field-dependant map [1,2, 4, 5, 7, 10, After such a mapping it is seen that the hamiltonian symmetries indeed give back the Poincaré symmetries, but modulo terms proportional to equations of motion [4,5].

$$
\delta_{G} b_{\mu}^{i} \sim \delta_{P G T} b_{\mu}^{i}+\text { Eqns. of motion }
$$

So the hamiltonian symmetries are not exactly equal to the Poincaré symmetries and it seems that we may have two independent sets of symmetries for the same action! Each of these symmetries will now give rise to their own independent Noether identities.

This is not a desirable situation. It leads to an increase in the total number of independent gauge parameters over and above that found through the canonical analysis. We now have to take the Poincaré symmetry parameters in addition to the hamiltonian gauge parameters, if they are distinct. Also, we have to deal with more number of independent Noether identities than the number of Poncaré symmetries present in the model. But we know that the number of gauge parameters and Noether identities must match the total number of independent, primary, first-class constraints [16-18]. This creates an apparent paradoxical situation.

In this article we provide a resolution of this paradox by pointing out that the pair of symmetries differ only through trivial gauge transformations. These types of transformations [11] are not generated by first-class constraints of the theory. Thus they do not introduce any new arbitrary functions of time, i.e. they give rise to no new gauge parameters. Hence they indicate no degeneracy in the equations of motion and their solutions, representing physical states, are not mapped to new degenerate states through these transformations. Thus trivial gauge symmetries are not physical and the hamiltonian mechanism actually reproduce the Poinacré symmetries as the only physically relevant symmetries of the theory. Such symmetries also produce no new independent Noether identity and so the total number of identities and gauge parameters match the original number of Poincaré symmetries. By exploiting the Noether identities we provide a method to construct the 
map between the hamiltonian and Poincaré gauge parameters. Finally, through this work, explicit examples of trivial gauge symmetries and the role they play in many well studied field theories get highlighted.

We now explain the organisation of our article. In section 2 we give a brief overview of trivial gauge symmetries from a general point of view, including a discussion on their role in the Noether identities. In section 3, we take up the first order generalisation of Einstein gravity - the Einstein-Cartan model. We explicitly show how the two sets of symmetries and Noether identities corresponding to hamiltonian and PGT formulations are related via trivial gauge transformations. We also motivate an algorithm to find suitable map between gauge parameters to enable a comparison between these two sets of symmetries. In section 4 , the analysis is performed in a generalisation of the previous model to a Mielke-Baekler type gravity [19,20] extended by a cosmological term. This provides a further non-trivial demonstration of our results. Finally, we conclude in section 5 ,

Summary of conventions: Latin indices refer to the local Lorentz frame and the Greek indices refer to the coordinate frame. The beginning letters of both alphabets $(a, b, c, \ldots)$ and $(\alpha, \beta, \gamma, \ldots)$ run over the space part $(1,2)$ while the middle alphabet letters $(i, j, k, \ldots)$ and $(\mu, \nu, \lambda, \ldots)$ run over all coordinates $(0,1,2)$. The totally antisymmetric tensor $\epsilon^{i j k}$ and the tensor density $\epsilon^{\mu \nu \rho}$ are both normalized so that $\epsilon^{012}=1$. The signature of space-time adopted here is $\eta=\operatorname{diag}(+,-,-)$.

\section{Gauge symmetries and trivial gauge symmetries}

Let $S\left[q_{i}\right]$ describe an action with the basic field variables being $q_{i}(i=1,2, \ldots, n)$. The canonical momenta are then defined as $\pi^{i}=\frac{\delta S}{\delta \dot{q}_{i}}$ and the hamiltonian phase space is constructed out of the conjugate pair $\left(q_{i}, \pi^{i}\right)$. The standard canonical procedure [21] yields all the constraints. Let us denote the first class constraints as $\Sigma_{a},(a=1,2, \ldots, f)$ and the second class constraints as $\chi_{b}$ $(b=1,2, \ldots, s)$, with $P=f+s$ being the total number of constraints. The Dirac prescription gives the gauge generator as a linear combination of all first class constraints

$$
G=\alpha^{a} \Sigma_{a},
$$

$\alpha^{a}$ 's being arbitrary parameters in time. However, not all the parameters $\alpha^{a}$ are independent. We can eliminate the dependant ones systematically and write the gauge generator in terms of only the independent $\alpha^{a}$ 's, following a completely off-shell method [16-18] 1] The final generator yields the gauge transformations of fields through a Poisson bracket 2 operation with the fields. There exist two different possibilities of defining this operation $\{q, G\}$, results being equivalent upto terms proportional in constraints.

$$
\begin{aligned}
\delta_{1} q & =\left\{q, \alpha^{a} \Sigma_{a}\right\} \\
\text { or, } \quad \delta_{2} q & =\alpha^{a}\left\{q, \Sigma_{a}\right\} .
\end{aligned}
$$

These two definitions of gauge transformations $\delta_{1}$ and $\delta_{2}$ differ upto 'trivial gauge transformations' [16].

Trivial gauge transformations keep the action invariant simply by a specific antisymmetric structure within them. To write explicitly, let us consider transformations of the form

$$
\delta q_{i}=\Lambda_{i j} \frac{\delta S}{\delta q_{j}}, \quad \Lambda_{i j}=-\Lambda_{j i}
$$

\footnotetext{
${ }^{1}$ There are other methods of construction of a gauge generator like [22, though it is not an off-shell one.

${ }^{2}$ Or a Dirac bracket, if the second class sector has been eliminated through introduction of Dirac brackets.
} 
Here $\frac{\delta S}{\delta q_{j}}$ is the Euler derivative corresponding to the field $q_{j}$ and its equation of motion is given by setting this Euler derivative to zero. Thus on-shell, i.e. after imposition of equations of motion, trivial gauge transformations vanish. However invariance of the action $(\delta S=0)$ is achieved off-shell due to the antisymmetry of $\Lambda_{i j}$

$$
\begin{aligned}
\delta S & =\frac{\delta S}{\delta q_{i}} \delta q_{i} \\
& =\frac{\delta S}{\delta q_{i}} \Lambda_{i j} \frac{\delta S}{\delta q_{j}}=0,
\end{aligned}
$$

as the product $\frac{\delta S}{\delta q_{i}} \frac{\delta S}{\delta q_{j}}$ is symmetric in $i \& j$. Since these transformations vanish on-shell they imply no degeneracy in the solutions of the equations of motion; i.e. they do not map a set of solutions to any other set through arbitrary functions of time, unlike true gauge transformations. Given any action, they can always be added as symmetry transformations and the specific form of the co-efficients do not matter, as long as they are antisymmetric in the field indices. They are not generated by first-class constraints in the hamiltonian formalism and give rise to zero gauge current as they are on-shell symmetries. Thus, trivial gauge symmetries are not true gauge symmetries and are of no physical importance.

As a consequence of the above discussion, it can be anticipated that the trivial gauge symmetries do not give rise to any new Noether identities, other than those already present due to the true symmetries of the system. Given any gauge symmetry parametrised by an arbitrary time function $\sigma$ (known as the gauge parameter),

$$
\delta q_{i}=R_{i \mu} \sigma^{\mu}+\tilde{R}_{i \mu}{ }^{\nu} \partial_{\nu} \sigma^{\mu}
$$

where $R_{i}$ 's and $\tilde{R}_{i}$ 's are functions of the fields $q_{i}$ and possibly their derivatives, the invariance of the action leads to

$$
\begin{aligned}
\delta S & =\int \frac{\delta \mathcal{L}}{\delta q_{i}} \delta q_{i} \\
& =\int \frac{\delta \mathcal{L}}{\delta q_{i}}\left(R_{i \mu} \sigma^{\mu}+\tilde{R}_{i \mu}{ }^{\nu} \partial_{\nu} \sigma^{\mu}\right) \\
& =\int\left[\frac{\delta \mathcal{L}}{\delta q_{i}} R_{i \mu}-\partial_{\nu}\left(\frac{\delta \mathcal{L}}{\delta q_{i}} \tilde{R}_{i \mu}{ }^{\nu}\right)\right] \sigma^{\mu}=0 .
\end{aligned}
$$

Since $\sigma$ is an arbitrary function, we can write

$$
\frac{\delta \mathcal{L}}{\delta q_{i}} R_{i \mu}-\partial_{\nu}\left(\frac{\delta \mathcal{L}}{\delta q_{i}} \tilde{R}_{i \mu}{ }^{\nu}\right)=0
$$

which are the Noether identities of the system 3 . They imply a dependence of the Euler derivatives $\frac{\delta \mathcal{L}}{\delta q_{i}}$ among themselves and thus the equations of motion are not all independent. Note that each Noether identity is proportional to a gauge parameter (here $\sigma^{\mu}$ ). Thus combinations of one set of independent Noether identities among themselves to give rise to another equivalent set of identities is reflected at the symmetry level as a redefinition of the old gauge parameters into a new set of gauge parameters.

\footnotetext{
${ }^{3}$ For a lagrangian analysis based on these identities, in the context of the Mielke-Baekler model, see [23].
} 
Now trivial gauge symmetries may affect the Noether identities in many ways. In a direct manner, if $R_{i}$ has antisymmetric contributions like

$$
R_{i \mu} \rightarrow R_{i \mu}+\left(\Lambda_{i j}\right)_{\mu} \frac{\delta \mathcal{L}}{\delta q_{j}} \quad\left(\Lambda_{i j}\right)_{\mu}=-\left(\Lambda_{j i}\right)_{\mu},
$$

as can arise from transformations like (2), then we will have extensions of the gauge identities (5) as shown below

$$
\frac{\delta \mathcal{L}}{\delta q_{i}} R_{i \mu}-\partial_{\nu}\left(\frac{\delta \mathcal{L}}{\delta q_{i}} \tilde{R}_{i \mu}{ }^{\nu}\right)+\frac{\delta \mathcal{L}}{\delta q_{i}}\left(\Lambda_{i j}\right)_{\mu} \frac{\delta \mathcal{L}}{\delta q_{j}}=0
$$

However the last term vanishes by itself, without depending on the particular structure of the Euler derivatives, through (anti)symmetry. This generates no new identities and thus the Noether identities (5) and (6) are infact equivalent to each other and correspond to only one physical symmetry.

In the following sections, we work with explicit models (Einstein-Cartan gravity and a MielkeBaekler [19,20] type gravity) to show the role of trivial gauge symmetries in relating hamiltonian symmetries to the Poincaré symmetries. The analysis in each case will be based on the general formalism outlined in this section.

\section{Einstein - Cartan gravity}

The Einstein-Cartan formulation of gravity is a first order generalisation of Einstein's general relativity. It is constructed through a Poincaré gauge theory (PGT) construction, 2, 12,15] on a Riemann-Cartan spacetime having both curvature, as well as torsion. To start with, triad fields $b^{i}{ }_{\mu}(x)$ are set up at each point of spacetime to translate between local coordinates $x^{i}$ and global coordinates $x^{\mu}$. Thus, for any vector $A_{\mu}$, we have $A_{\mu}=b_{\mu}^{i} A_{i}$. The global metric $g_{\mu \nu}$ is written in terms of the triads and the local flat Minkowski metric $\eta^{i j}$ as

$$
g_{\mu \nu}=b_{\mu}^{i} b_{\nu}^{j} \eta_{i j}
$$

At this stage, there is a global Poincaré symmetry parametrised by Lorentz rotations and translations. To localise this Poincaré symmetry, covariant derivatives are brought in to replace partial derivatives and spin-connection fields $\omega_{\mu}^{i}$ are introduced. These comprise independent fields in PGT. The corresponding field strengths that come into play through the commutators of covariant derivatives give rise to torsion $T^{i}{ }_{\mu \nu}$ and curvature $R_{\mu \nu}^{i}$ tensors. Their forms turn out to be

$$
\begin{aligned}
R_{\mu \nu}^{i} & =\partial_{\mu} \omega^{i}{ }_{\nu}-\partial_{\mu} \omega^{i}{ }_{\nu}+\epsilon^{i}{ }_{j k} \omega^{j}{ }_{\mu} \omega^{k}{ }_{\nu} \\
T^{i}{ }_{\mu \nu} & =\nabla_{\mu} b^{i}{ }_{\nu}-\nabla_{\nu} b^{i}{ }_{\mu} .
\end{aligned}
$$

Here the covariant derivative of the triad is defined as $\nabla_{\mu} b^{i}{ }_{\nu}=\partial_{\mu} b^{i}{ }_{\nu}+\epsilon^{i}{ }_{j k} \omega^{j}{ }_{\mu} b^{k}{ }_{\nu}$. The PGT gravity models are constructed to be invariant under the local Poincaré transformations

$$
\begin{aligned}
\delta_{P G T} b^{i}{ }_{\mu} & =-\epsilon_{j k}^{i} b_{\mu}^{j} \theta^{k}-\partial_{\mu} \xi^{\rho} b^{i}{ }_{\rho}-\xi^{\rho} \partial_{\rho} b^{i}{ }_{\mu} \\
\delta_{P G T} \omega^{i}{ }_{\mu} & =-\partial_{\mu} \theta^{i}-\epsilon_{j k}^{i} \omega_{\mu}^{j} \theta^{k}-\partial_{\mu} \xi^{\rho} \omega_{\rho}^{i}-\xi^{\rho} \partial_{\rho} \omega^{i}{ }_{\mu} .
\end{aligned}
$$

In the above symmetries, the parameter describing local Lorentz transformations is $\theta^{i}$ and that describing general coordinate transformations is $\xi^{\mu}$ (both transformations being of infinitesimal 
order). Intuitively, this explains the structure of the transformations (8) where the index ' $i$ ' transforms as a Lorentz index while ' $\rho$ ' transforms as a general coordinate index. 4 The number of independent Poincaré symmetries for each field $(b, \omega$, or any other field, if present) is reflected in the number of independent gauge parameters. In our model in $3 \mathrm{D}, i=0,1,2$ and $\rho=0,1,2$. So the total number is

$$
3 \text { against } \xi^{\rho}+3 \text { against } \theta^{i}=6 .
$$

So we expect to find 6 independent gauge parameters and 6 independent Noether identities in our model and no more.

The Einstein-Cartan theory in Riemann-Cartan spacetime gives back the standard Einstein gravity on imposition of the zero torsion condition. The action, in $3 \mathrm{D}$, is

$$
S=\int \mathrm{d}^{3} \mathrm{x} a \epsilon^{\mu \nu \rho} b_{\mu}^{i} R_{i \nu \rho} .
$$

The basic variables of the theory are $b^{i}{ }_{\mu}$ and $\omega^{i}{ }_{\mu}$ with the corresponding conjugate momenta being denoted by $\pi_{i}{ }^{\mu}$ and $\Pi_{i}{ }^{\mu}$ respectively. The variational equations of motion are given by setting the Euler derivatives $\frac{\delta S}{\delta b^{i}}$ and $\frac{\delta S}{\delta \omega^{i}{ }_{\mu}}$ to zero.

$$
\begin{aligned}
& \frac{\delta S}{\delta b^{i}{ }_{\mu}}=a \epsilon^{\mu \nu \rho} R_{i \nu \rho}=0 \\
& \frac{\delta S}{\delta \omega^{i}{ }_{\mu}}=a \epsilon^{\mu \nu \rho} T_{i \nu \rho}=0
\end{aligned}
$$

A Dirac canonical analysis leads to the constraint structure [4,5] as given in Table 1, The relevant

\begin{tabular}{l|cc}
\hline \hline & First Class & Second class \\
\hline Primary & $\phi_{i}{ }^{0}, \Phi_{i}{ }^{0}$ & $\phi_{i}{ }^{\alpha}, \Phi_{i}{ }^{\alpha}$ \\
Secondary & $\overline{\mathcal{H}}_{i}, \overline{\mathcal{K}}_{i}$ & \\
\hline \hline
\end{tabular}

Table 1: Constraints of the EC theory.

quantities in Table 1 are defined below:

$$
\begin{aligned}
\phi_{i}{ }^{\mu} & =\pi_{i}{ }^{\mu} \\
\Phi_{i}{ }^{\mu} & =\Pi_{i}{ }^{\mu}-2 a \epsilon^{0 \alpha \beta} b_{i \beta} \delta_{\alpha}^{\mu} \\
\overline{\mathcal{H}}_{i} & =\left[-a \epsilon^{0 \alpha \beta} R_{i \alpha \beta}\right]-\nabla_{\alpha} \phi_{i}{ }^{\alpha} \\
\overline{\mathcal{K}}_{i} & =\left[-a \epsilon^{0 \alpha \beta} T_{i \alpha \beta}\right]-\nabla_{\alpha} \Phi_{i}{ }^{\alpha}-\epsilon_{i j k} b_{\alpha}{ }_{\alpha} \phi^{k \alpha}
\end{aligned}
$$

Once we have the constraints, we can construct the generator through an explicitly off-shell method [17, 18]. For Einstein-Cartan gravity, it turns out to be [5]

$$
\begin{aligned}
G= & \dot{\varepsilon}^{i} \pi_{i}{ }^{0}+\varepsilon^{i}\left[\overline{\mathcal{H}}_{i}-\epsilon_{i j k} \omega_{0}^{j} \pi^{k 0}\right] \\
& +\dot{\tau}^{i} \Pi_{i}{ }^{0}+\tau^{i}\left[\overline{\mathcal{K}}_{i}-\epsilon_{i j k}\left(b_{0}^{j} \pi^{k 0}+\omega_{0}^{j} \Pi^{k 0}\right)\right] .
\end{aligned}
$$

\footnotetext{
${ }^{4}$ For a more detailed discussion one may refer to [4,5].
} 
The hamiltonian gauge symmetries are calculated from the generator $G$, adopting the second among the definitions in (11)

$$
\begin{aligned}
\delta_{G} b_{\mu}^{i} & =\nabla_{\mu} \varepsilon^{i}+\epsilon_{j k}^{i} b_{\mu}{ }_{\mu} \tau^{k} \\
\delta_{G} \omega^{i}{ }_{\mu} & =\nabla_{\mu} \tau^{i} .
\end{aligned}
$$

Now the generator (13) is constructed as a linear combination of the products of first class constraints with gauge parameters. Looking at the first-class constraints in Table 1, we see that they all have one local index as their free-index. This fixes the structure of the gauge parameters $\varepsilon^{i}$ and $\tau^{i}$ in the hamiltonian formulation and they turn out to be different from the Poincaré gauge parameters $\xi^{\rho}$ and $\theta^{i}$, translations and local Lorentz rotations, seen in (8). However, to compare between the two symmetries $\delta_{G}$ and $\delta_{P G T}$ we must first have structurally similar set of gauge parameters in both sets of symmetries. This is achieved by introducing a field dependant map between the hamiltonian and Poincaré gauge parameters [2,4,5]

$$
\varepsilon^{i}=-\xi^{\rho} b_{\rho}^{i} \quad \& \quad \tau^{i}=-\theta^{i}-\xi^{\rho} \omega_{\rho}^{i} .
$$

But this map is usually proposed arbitrarily and there is no process to generate this map from physical considerations. Using this map in the symmetries (14) and after a bit of manipulations, we arrive at

$$
\begin{aligned}
\delta_{G} b_{\mu}^{i} & =\delta_{P G T} b^{i}{ }_{\mu}+\frac{1}{2 a} \xi^{\rho} \epsilon_{\mu \nu \rho} \frac{\delta S}{\delta \omega_{i \nu}} \\
\delta_{G} \omega^{i}{ }_{\mu} & =\delta_{P G T} \omega^{i}{ }_{\mu}+\frac{1}{2 a} \xi^{\rho} \epsilon_{\mu \nu \rho} \frac{\delta S}{\delta b_{i \nu}},
\end{aligned}
$$

where the Euler derivatives are defined in (11). So the two sets of symmetries are different, and match only on-shell. Consequently, they also give rise to two sets of Noether identities.

The Noether identities corresponding to the PGT symmetries (8) can be found by proceeding along the route leading to (5). Explicitly, they are [5]

$$
\begin{aligned}
& P_{k}=\frac{\delta S}{\delta b^{i}{ }_{\mu}} \varepsilon^{i}{ }_{j k} b_{\mu}^{j}+\frac{\delta S}{\delta \omega^{i}{ }_{\mu}} \varepsilon^{i}{ }_{j k} \omega^{j}{ }_{\mu}-\partial_{\mu}\left(\frac{\delta S}{\delta \omega^{k}{ }_{\mu}}\right)=0 \\
& R_{\rho}=\frac{\delta S}{\delta b^{i}{ }_{\mu}} \partial_{\rho} b^{i}{ }_{\mu}+\frac{\delta S}{\delta \omega^{i}{ }_{\mu}} \partial_{\rho} \omega_{\mu}^{i}-\partial_{\mu}\left(b_{\rho}^{i} \frac{\delta S}{\delta b^{i}{ }_{\mu}}+\omega^{i}{ }_{\rho} \frac{\delta S}{\delta \omega^{i}{ }_{\mu}}\right)=0 .
\end{aligned}
$$

The total number is $3+3=6$, as expected. Those corresponding to the hamiltonian gauge transformations (14) are, similarily,

$$
\begin{aligned}
& A_{k}=-\partial_{\mu}\left(\frac{\delta S}{\delta \omega^{k}{ }_{\mu}}\right)+\frac{\delta S}{\delta b^{i}{ }_{\mu}} \varepsilon^{i}{ }_{j k} b_{\mu}^{j}+\frac{\delta S}{\delta \omega^{i}{ }_{\mu}} \varepsilon^{i}{ }_{j k} \omega^{j}{ }_{\mu}=0 \\
& B_{k}=-\partial_{\mu}\left(\frac{\delta S}{\delta b^{k}{ }_{\mu}}\right)+\frac{\delta S}{\delta b^{i}{ }_{\mu} \varepsilon^{i}{ }_{j k} \omega^{j}{ }_{\mu}=0}
\end{aligned}
$$

and are also $3+3=6$ in number. We would like to emphasise at this point that these identities are to be dealt with off-shell, without imposition of equations of motion, i.e. without setting the Euler derivatives to be zero. The identities in-fact become tautological $0=0$ statements on-shell as they are comprised of the Euler derivatives.

Now the question that we want to address is whether the sets of identities (17) and (18) are independent, or can they be shown to be actually the same. A comparison shows that among the 
two sets, (17a) and (18a) are already identical, i.e. $P_{k} \equiv A_{k}$. We want to check the possibility of expressing $R_{\rho}$ as some linear combination of $P_{k}$ and $R_{k}$. Comparing the structure of the free indices and the derivative terms among (17a) and (18a) we see that the combination $-b^{k}{ }_{\rho} B_{k}-\omega_{\rho}^{k} A_{k}$ gives us

$$
-b_{\rho}^{k} B_{k}-\omega^{k}{ }_{\rho} A_{k}=-R_{\rho}+\frac{\delta S}{\delta b^{i}{ }_{\mu}}\left(\frac{1}{2 a} \eta^{i j} \epsilon_{\mu \nu \rho}\right) \frac{\delta S}{\delta \omega^{j}{ }_{\nu}}+\frac{\delta S}{\delta \omega^{i}{ }_{\mu}}\left(\frac{1}{2 a} \eta^{i j} \epsilon_{\mu \nu \rho}\right) \frac{\delta S}{\delta b^{j}{ }_{\nu}}=0
$$

The last two terms in the above equation, proportional to square of Euler derivatives, cancel out due to antisymmetry of their coefficients without requiring the particular form of the Euler derivatives (11). The net identity obtained in the process is just the second Noether identity corresponding to the Poincaré symmetries. Thus we show that there exists only one set of true, independent Noether identities in the system and the total number of these are $3+3=6$, i.e. equal to the total number of gauge symmetries in the system.

The Noether identities are obtained, as shown in (4) and (5), from collecting coefficients of the independent gauge parameters from a variation of the action through functional Taylor expansion

$$
\begin{aligned}
\delta S & =\int\left(\theta^{k} P_{k}+\xi^{\rho} R_{\rho}\right)=0 & \text { Poincaré symmetries. } \\
\delta S & =\int\left(\varepsilon^{k} A_{k}+\tau^{k} B_{k}\right)=0 & \text { hamiltonian symmetries. }
\end{aligned}
$$

The combinations $R_{\rho}=-b_{\rho}^{k} B_{k}-\omega^{k}{ }_{\rho} A_{k}$ and $P_{k}=-A_{k}$, when substituted in (20), gives

$$
\int\left[\left(-\theta^{k}-\xi^{\rho} \omega_{\rho}^{k}\right) A_{k}+\left(-b_{\rho}^{k} \xi^{\rho}\right) B_{k}\right]=0 .
$$

Comparing this with (21) gives us the required map (15) between the two sets of gauge parameters. So the Noether identities help us to generate the required map between different sets of gauge parameters.

It is desirable to point out that, in the above analysis, we have not used any connection between the Noether identities and equations of motion. A literal application of the dependence of EulerLagrange equations due to Noether identities, mentioned below (5), may lead to incorrect results 5 Here we have compared the Noether identities arising from the PGT and hamiltonian approaches to motivate the map (15). Also, all the Noether identities were explicitly verified.

The structure of the antisymmetric terms obtained in (19), when compared with those that arise in the case of trivial gauge symmetries as outlined in (6), hints at the presence of trivial gauge symmetries within the hamiltonian formalism. The general form of trivial gauge transformations in this model would read

$$
\begin{aligned}
\delta b^{i}{ }_{\mu} & =\Lambda_{\left(b^{i}{ }_{\mu}, b^{j}{ }_{\nu}\right)} \frac{\delta S}{\delta b^{j}{ }_{\nu}}+\Lambda_{\left(b^{i}{ }_{\mu}, \omega^{j}{ }_{\nu}\right)} \frac{\delta S}{\delta \omega^{j}{ }_{\nu}} \\
\delta \omega^{i}{ }_{\mu} & =\Lambda_{\left(\omega^{i}{ }_{\mu}, b^{j}{ }_{\nu}\right)} \frac{\delta S}{\delta b^{j}{ }_{\nu}}+\Lambda_{\left(\omega^{i}{ }_{\mu}, \omega^{j}{ }_{\nu}\right)} \frac{\delta S}{\delta \omega^{j}{ }_{\nu}}
\end{aligned}
$$

where $\Lambda$ is antisymmetric (see (2)). Here $\delta \equiv \delta_{G}-\delta_{P G T}$ is the apparently extra symmetry present within the hamiltonian symmetries. Comparing this with (16) we find the $\Lambda$ matrix defining the

\footnotetext{
${ }^{5}$ This point was brought to our notice by the referee who also suggested, in this context, the original classic works of Hilbert on general relativity.
} 
trivial gauge symmetry to be

$$
\begin{aligned}
\Lambda_{\left(b^{i}{ }_{\mu}, b^{j}{ }_{\nu}\right)} & =0 & \Lambda_{\left(b^{i}{ }_{\mu}, \omega^{j}{ }_{\nu}\right)} & =\frac{1}{2 a} \eta^{i j} \xi^{\rho} \epsilon_{\mu \nu \rho} \\
\Lambda_{\left(\omega^{i}{ }_{\mu}, b^{j}{ }_{\nu}\right)} & =\frac{1}{2 a} \eta^{i j} \xi^{\rho} \epsilon_{\mu \nu \rho} & \Lambda_{\left(\omega^{i}{ }_{\mu}, \omega^{j}{ }_{\nu}\right)} & =0
\end{aligned}
$$

The antisymmetry of $\Lambda$ in the diagonal $(b-b$ or $\omega-\omega)$ entries is obvious. For the off-diagonal entry,

$$
\begin{aligned}
\Lambda_{\left(b^{i}{ }_{\mu}, \omega^{j}{ }_{\nu}\right)} & =\frac{1}{2 a} \eta^{i j} \xi^{\rho} \epsilon_{\mu \nu \rho} \\
& =-\frac{1}{2 a} \eta^{j i} \xi^{\rho} \epsilon_{\nu \mu \rho} \\
& =-\Lambda_{\left(\omega^{j}{ }_{\nu}, b^{i}{ }_{\mu}\right)} .
\end{aligned}
$$

Thus the $\Lambda$ matrix is antisymmetric in its field indices and this renders the action off-shell invariant. We have thus shown that the difference between the hamiltonian and Poincaré symmetries is just a trivial gauge transformation. The total number of true physical symmetries remain $3+3=6$ as both $\delta_{G}$ and $\delta_{P G T}$ are now physically equivalent.

\section{$43 \mathrm{D}$ cosmological gravity with torsion}

In this section, we study a 3D gravity model based on the Mielke-Baekler (MB) action [19, 20, added with a cosmological term. This is formulated with triad - spin-connection variables, in the PGT formalism. The canonical analysis of this model done in [4] shows the same feature of hamiltonian and Poincaré gauge symmetries being related, modulo on-shell vanishing terms.

The action describing this topological 3D gravity model with torsion and a cosmological term is

$$
S=\int \mathrm{d}^{3} \mathrm{x} \epsilon^{\mu \nu \rho}\left[a b^{i}{ }_{\mu} R_{i \nu \rho}-\frac{\Lambda}{3} \epsilon_{i j k} b_{\mu}^{i} b_{\nu}^{j} b_{\rho}^{k}+\alpha_{3}\left(\omega^{i}{ }_{\mu} \partial_{\nu} \omega_{i \rho}+\frac{1}{3} \epsilon_{i j k} \omega^{i}{ }_{\mu} \omega^{j}{ }_{\nu} \omega^{k}{ }_{\rho}\right)+\frac{\alpha_{4}}{2} b^{i}{ }_{\mu} T_{i \nu \rho}\right]
$$

Basic variables here are $b^{i}{ }_{\mu}$ and $\omega^{i}{ }_{\mu}$ and corresponding momenta are denoted $\pi_{i}{ }^{\mu}$ and $\Pi_{i}{ }^{\mu}$ respectively. The equations of motion are obtained by setting to zero the various Euler derivatives,

$$
\begin{gathered}
\frac{\delta S}{\delta b^{i}{ }_{\mu}}=\epsilon^{\mu \nu \rho}\left[a R_{i \nu \rho}+\alpha_{4} T_{i \nu \rho}-\Lambda \epsilon_{i j k} b^{j}{ }_{\nu} b_{\rho}^{k}\right]=0 \\
\frac{\delta S}{\delta \omega^{i}{ }_{\mu}}=\epsilon^{\mu \nu \rho}\left[\alpha_{3} R_{i \nu \rho}+a T_{i \nu \rho}+\alpha_{4} \epsilon_{i j k} b^{j}{ }_{\nu} b_{\rho}^{k}\right]=0
\end{gathered}
$$

All the momenta turn out to be primary constraints in this first order theory. The consistency process ends at the secondary level itself and the constraints can be classified [7] as given in Table (2). The relevant quantities used are defined below: 


\begin{tabular}{l|cc}
\hline \hline & First Class & Second class \\
\hline Primary & $\phi_{i}{ }^{0}, \Phi_{i}{ }^{0}$ & $\phi_{i}{ }^{\alpha}, \Phi_{i}{ }^{\alpha}$ \\
Secondary & $\overline{\mathcal{H}}_{i}, \overline{\mathcal{K}}_{i}$ & \\
\hline \hline
\end{tabular}

Table 2: Constraints of the MB type 3D gravity theory.

$$
\begin{aligned}
\phi_{i}{ }^{\mu} & =\pi_{i}{ }^{\mu}-\alpha_{4} \epsilon^{0 \alpha \beta} b_{i \beta} \delta_{\alpha}^{\mu} \\
\Phi_{i}{ }^{\mu} & =\Pi_{i}{ }^{\mu}-\epsilon^{0 \alpha \beta}\left(2 a b_{i \beta}+\alpha_{3} \omega_{i \beta}\right) \delta_{\alpha}^{\mu} \\
\overline{\mathcal{H}}_{i} & =-\left[\epsilon^{0 \alpha \beta}\left(a R_{i \alpha \beta}+\alpha_{4} T_{i \alpha \beta}-\Lambda \epsilon_{i j k} b_{\alpha}{ }_{\alpha} b_{\beta}^{k}\right)\right]-\nabla_{\alpha}{\phi_{i}}^{\alpha}+\epsilon_{i j k} b_{\alpha}^{j}\left(p \phi^{k \alpha}+q \Phi^{k \alpha}\right) \\
\overline{\mathcal{K}}_{i} & =-\left[\epsilon^{0 \alpha \beta}\left(a T_{i \alpha \beta}+\alpha_{3} R_{i \alpha \beta}+\alpha_{4} \epsilon_{i j k} b^{j}{ }_{\alpha} b^{k}\right)\right]-\nabla_{\alpha} \Phi_{i}{ }^{\alpha}-\epsilon_{i j k} b_{\alpha}{ }_{\alpha} \phi^{k \alpha} \\
p & =\frac{\alpha_{3} \Lambda+\alpha_{4} a}{\alpha_{3} \alpha_{4}-a^{2}} ; \quad q=-\frac{\alpha_{4}^{2}+a \Lambda}{\alpha_{3} \alpha_{4}-a^{2}}
\end{aligned}
$$

Here the terms within square brackets in the definitions of the constraints $\overline{\mathcal{H}}_{i}$ and $\overline{\mathcal{K}}_{i}$, are themselves secondary in nature. The classified constraints in Table (2) are suitable combinations of the primary and secondary constraints.

Using these constraints and an explicitly off-shell method [17, 18], the hamiltonian generator of gauge symmetries can be constructed [5]. There are two (indexed) gauge parameters $\varepsilon^{i}$ and $\tau^{i}$ and they are (again) different from the Poincaré gauge parameters $\xi^{\rho}$ and $\theta^{k}$. The generator ' $G$ ' can be written as a sum of two parts $-\mathcal{G}_{\varepsilon}$ and $\mathcal{G}_{\tau}$, as shown below

$$
\begin{aligned}
G= & \int d^{2} x\left[\mathcal{G}_{\varepsilon}(x)+\mathcal{G}_{\tau}(x)\right] \\
& \mathcal{G}_{\varepsilon}=\dot{\varepsilon}^{i} \pi_{i}{ }^{0}+\varepsilon^{i}\left[\overline{\mathcal{H}}_{i}-\varepsilon_{i j k}\left(\omega^{j}{ }_{0}-p b^{j}{ }_{0}\right) \pi^{k 0}+q \varepsilon_{i j k} b_{0}^{j} \Pi^{k 0}\right] \\
& \mathcal{G}_{\tau}=\dot{\tau}^{i} \Pi_{i}{ }^{0}+\tau^{i}\left[\overline{\mathcal{K}}_{i}-\varepsilon_{i j k}\left(b^{j}{ }_{0} \pi^{k 0}+\omega^{j}{ }_{0} \Pi^{k 0}\right)\right]
\end{aligned}
$$

Symmetries of the basic fields can be computed from this generator through the second definition among (11)

$$
\begin{aligned}
\delta b^{i}{ }_{\mu} & =\nabla_{\mu} \varepsilon^{i}-p \epsilon_{j k}^{i} b_{\mu}{ }_{\mu} \varepsilon^{k}+\epsilon^{i}{ }_{j k} b_{\mu}^{j} \tau^{k}, \\
\delta \omega^{i}{ }_{\mu} & =\nabla_{\mu} \tau^{i}-q \epsilon_{j k}^{i} b_{\mu}{ }_{\mu} \varepsilon^{k} .
\end{aligned}
$$

The hamiltonian symmetries contain the coupling constants $\Lambda, \alpha_{3}$ and $\alpha_{4}$ through the parameters $p \& q$ defined earlier. These, they inherit from the action through the structure of the constraints. To compare with Poincaré symmetries, we take recourse to the map (15) relating the hamiltonian gauge parameters to the Poincaré gauge parameters. After some rearrangements and remembering the Euler derivatives from (26), we arrive at

$$
\begin{aligned}
\delta_{G} b_{\mu}^{i} & =\delta_{P G T} b_{\mu}^{i}+\frac{\alpha_{3}}{2\left(\alpha_{3} \alpha_{4}-a^{2}\right)} \eta^{i j} \xi^{\rho} \epsilon_{\mu \nu \rho} \frac{\delta S}{\delta b^{j}{ }_{\nu}}-\frac{a}{2\left(\alpha_{3} \alpha_{4}-a^{2}\right)} \eta^{i j} \xi^{\rho} \epsilon_{\mu \nu \rho} \frac{\delta S}{\delta \omega^{j}{ }_{\nu}} \\
\delta_{G} \omega^{i}{ }_{\mu} & =\delta_{P G T} \omega^{i}{ }_{\mu}-\frac{a}{2\left(\alpha_{3} \alpha_{4}-a^{2}\right)} \eta^{i j} \xi^{\rho} \epsilon_{\mu \nu \rho} \frac{\delta S}{\delta b^{j}{ }_{\nu}}+\frac{\alpha_{4}}{2\left(\alpha_{3} \alpha_{4}-a^{2}\right)} \eta^{i j} \xi^{\rho} \epsilon_{\mu \nu \rho} \frac{\delta S}{\delta \omega^{j}{ }_{\nu}}
\end{aligned}
$$

It is again clear that the hamiltonian and Poincaré symmetries become identical only on-shell. 
Let us now investigate the Noether identities in this model. The identities corresponding to the PGT symmetries remain the same as (17), since the form of the Poincare symmetries do not depend upon the form of the lagrangian, as long as the lagrangian is diffeomorphism invariant in nature (and contains the same fields in construction of the action). The hamiltonian gauge symmetries (29) give rise to the following identities [5]

$$
\begin{aligned}
& A_{k}^{\prime}=-\partial_{\mu}\left(\frac{\delta S}{\delta \omega^{k}}\right)+\frac{\delta S}{\delta b^{i}{ }_{\mu}} \varepsilon^{i}{ }_{j k} b^{j}{ }_{\mu}+\frac{\delta S}{\delta \omega^{i}{ }_{\mu}} \varepsilon^{i}{ }_{j k} \omega^{j}{ }_{\mu}=0 \\
& B_{k}^{\prime}=-\partial_{\mu}\left(\frac{\delta S}{\delta b^{k}{ }_{\mu}}\right)+\frac{\delta S}{\delta b^{i}{ }_{\mu}} \varepsilon^{i}{ }_{j k} \omega^{j}{ }_{\mu}-p \frac{\delta S}{\delta b^{i}{ }_{\mu} \epsilon_{j k}{ }_{j k} b_{\mu}-q \frac{\delta S}{\delta \omega^{i}{ }_{\mu}} \epsilon^{i}{ }_{j k} b^{j}{ }_{\mu}=0 .}
\end{aligned}
$$

Once again we see that one of the identities among the hamiltonian gauge (31) and Poincaré ones (17), $A_{k}$ and $P_{k}$, match each other. And the combination $-\omega_{\rho}^{k} A_{k}^{\prime}+-b_{\rho}^{k} B_{k}^{\prime}$ leads to

$$
\begin{aligned}
-R_{\rho} & +\frac{\delta S}{\delta b^{i}{ }_{\mu}}\left(\frac{\alpha_{3}}{2\left(\alpha_{3} \alpha_{4}-a^{2}\right)} \eta^{i j} \epsilon_{\mu \nu \rho}\right) \frac{\delta S}{\delta b^{j}{ }_{\nu}}+\frac{\delta S}{\delta b^{i}{ }_{\mu}}\left(\frac{-a}{2\left(\alpha_{3} \alpha_{4}-a^{2}\right)} \eta^{i j} \epsilon_{\mu \nu \rho}\right) \frac{\delta S}{\delta \omega_{\nu}^{j}} \\
& +\frac{\delta S}{\delta \omega^{i}{ }_{\mu}}\left(\frac{-a}{2\left(\alpha_{3} \alpha_{4}-a^{2}\right)} \eta^{i j} \epsilon_{\mu \nu \rho}\right) \frac{\delta S}{\delta b^{j}{ }_{\nu}}+\frac{\delta S}{\delta \omega^{i}{ }_{\mu}}\left(\frac{\alpha_{4}}{2\left(\alpha_{3} \alpha_{4}-a^{2}\right)} \eta^{i j} \epsilon_{\mu \nu \rho}\right) \frac{\delta S}{\delta b^{j}{ }_{\nu}}=0 .
\end{aligned}
$$

The last four terms, proportional to square of Euler derivatives, cancel each other due to antisymmetry of their coefficients. The part surviving is just the missing Poincaré identity $R_{\rho}=0$ (17b). Thus there exist only one set of independent Noether identities.

The antisymmetric terms in the Noether identities (32) again point toward presence of trivial gauge symmetries. To check explicitly, we first write down the general trivial gauge symmetry structure appropriate for the MB model

$$
\begin{aligned}
\delta b^{i}{ }_{\mu} & =\Lambda_{\left(b^{i}{ }_{\mu}, b^{j}{ }_{\nu}\right)} \frac{\delta S}{\delta b^{j}{ }_{\nu}}+\Lambda_{\left(b^{i}{ }_{\mu}, \omega^{j}{ }_{\nu}\right)} \frac{\delta S}{\delta \omega^{j}{ }_{\nu}} \\
\delta \omega^{i}{ }_{\mu} & =\Lambda_{\left(\omega^{i}{ }_{\mu}, b^{j}{ }_{\nu}\right)} \frac{\delta S}{\delta b^{j}{ }_{\nu}}+\Lambda_{\left(\omega^{i}{ }_{\mu}, \omega^{j}{ }^{j}\right)} \frac{\delta S}{\delta \omega^{j}{ }_{\nu}}
\end{aligned}
$$

Comparing this with (30), we write can down the $\Lambda$ matrix below

$$
\begin{aligned}
\Lambda_{\left(b^{i}{ }_{\mu}, b^{j}{ }_{\nu}\right)} & =\frac{\alpha_{3}}{2\left(\alpha_{3} \alpha_{4}-a^{2}\right)} \eta^{i j} \xi^{\rho} \epsilon_{\mu \nu \rho} & \Lambda_{\left(b^{i}{ }_{\mu}, \omega^{j}{ }_{\nu}\right)} & =\frac{-a}{2\left(\alpha_{3} \alpha_{4}-a^{2}\right)} \eta^{i j} \xi^{\rho} \epsilon_{\mu \nu \rho} \\
\Lambda_{\left(\omega^{i}{ }_{\mu}, b^{j}{ }_{\nu}\right)} & =\frac{-a}{2\left(\alpha_{3} \alpha_{4}-a^{2}\right)} \eta^{i j} \xi^{\rho} \epsilon_{\mu \nu \rho} & \Lambda_{\left(\omega^{i}{ }_{\mu}, \omega^{j}{ }_{\nu}\right)} & =\frac{\alpha_{4}}{2\left(\alpha_{3} \alpha_{4}-a^{2}\right)} \eta^{i j} \xi^{\rho} \epsilon_{\mu \nu \rho}
\end{aligned}
$$

The antisymmetry of this structure is easy to verify. We will just demonstrate one component

$$
\begin{aligned}
\Lambda_{\left(b^{i}{ }_{\mu}, b_{\nu}{ }_{\nu}\right)} & =\frac{\alpha_{3}}{2\left(\alpha_{3} \alpha_{4}-a^{2}\right)} \eta^{i j} \xi^{\rho} \epsilon_{\mu \nu \rho} \\
& =-\frac{\alpha_{3}}{2\left(\alpha_{3} \alpha_{4}-a^{2}\right)} \eta^{j i} \xi^{\rho} \epsilon_{\nu \mu \rho} \\
& =-\Lambda_{\left(b^{j}{ }_{\nu}, b^{i}{ }_{\mu}\right)} .
\end{aligned}
$$

So the two symmetries $\delta_{G}$ and $\delta_{P G T}$ differ only by a trivial gauge symmetry which is of no physical importance. The Poincaré transformations are indeed recovered by the hamiltonian mechanism. An important point to be noted from the analysis of this model is that the hamiltonian symmetries (29) of this model were different from those of the Einstein-Cartan theory (14). However we could nevertheless recover the Poincaré symmetries from both of these. The particular difference in details between the models (various terms in the action along with their coupling constants) got manifested only through trivial gauge symmetries. 


\section{Discussions}

We have shown in this paper that the Dirac hamiltonian construction indeed reproduces the Poincaré symmetries in different models of gravity. We have analysed the Einstein-Cartan action and a more generalised form of a Mielke-Baekler type action with a cosmological term, both in 3dimensions. The Noether identities corresponding to the two sets of symmetries, hamiltonian gauge and Poincaré, were shown to be the same, modulo antisymmetric cancelling terms proportional to square of Euler derivatives. Using these Noether identities, we derived a map between the two sets of gauge parameters. After using the map, we demonstrated that the difference in the hamiltonian gauge symmetries and the Poincaré symmetries was just trivial gauge transformations, characterised by coefficients antisymmetric under exchange of fields. We have explicitly found out the coefficient matrices for both Einstein-Cartan and its Mielke-Baekler type generalisation.

Since trivial gauge symmetries are of no physical importance, the Poincaré symmetries are indeed recovered through the canonical procedure. This feature should persist in all the different diffeomorphism invariant theories of interest and shows the importance of understanding and handling trivial gauge symmetries.

We have shown how the lagrangian and hamiltonian formulations complement each other and how their unified application is of great importance. Analysis of the Noether identities arising in the lagrangian formulation helps us to construct the map between gauge parameters present in the hamiltonian and Poincaré gauge transformations. This map, at the hamiltonian level, can only be guessed through an (in general case, a rather difficult) exercise of inspection and trial. In the lagrangian procedure, however, the process is much more straightforward and systematic. It is noteworthy that the map is model independent, i.e. it is the same in both examples studied here. This universal nature reveals a unifying feature among the hamiltonian gauge symmetries, a fact that is not otherwise transparent. Indeed, contrary to Poincaré gauge transformations, the structure of hamiltonian gauge transformations are distinct for distinct models.

Finally, let us recall the role of trivial gauge transformations at the quantum level. This is relevant since gauge symmetries are important in the process of quantisation. The classical gauge symmetries of the action are now replaced by the quantum (Becchi-Rouet-Stora-Tyutin or BRST) symmetries of the quantum effective action $(\Gamma)$. For general gauge theories it was shown [24] that the set of local symmetries of $\Gamma$ comprise of the quantum gauge transformations, trivial gauge transformations and transformations induced by background fields. Taking a linear combination of all three symmetries, it is possible to find a simple or a standard form. Indeed, adopting this approach the classical gauge transformations for Yang Mills theory were reproduced in [24].

\section{References}

[1] E. Witten, "(2+1)-Dimensional Gravity as an Exactly Soluble System," Nucl. Phys. B311, 46 (1988).

[2] M. Blagojevic, "Gravitation and gauge symmetries," Bristol, UK: IOP (2002) and references therein.

[3] A. M. Frolov, N. Kiriushcheva, S. V. Kuzmin, "Hamiltonian formulation of tetrad gravity: Three dimensional case," Grav. Cosmol. 16, 181-194 (2010). [arXiv:0902.0856 [gr-qc]]. 
[4] M. Blagojevic and B. Cvetkovic, "Canonical structure of 3D gravity with torsion," Trends in GR and QC, 2, ed. Ch. Benton, NY: Nova Science (2006) 103-123 arXiv:gr-qc/0412134.

[5] R. Banerjee, S. Gangopadhyay, P. Mukherjee and D. Roy, "Symmetries of topological gravity with torsion in the hamiltonian and lagrangian formalisms," JHEP 1002, 075 (2010) arXiv:0912.1472 [gr-qc]].

[6] S. Deser, R. Jackiw and S. Templeton, "Topologically massive gauge theories," Annals Phys. 140, 372 (1982) [Erratum-ibid. 185, 406 (1988)]; Reprinted Annals Phys. 281, 409 (2000).

[7] M. Blagojevic and B. Cvetkovic, "Canonical structure of topologically massive gravity with a cosmological constant," JHEP 0905, 073 (2009) arXiv:0812.4742 [gr-qc]].

[8] M. Blagojevic and B. Cvetkovic, "Hamiltonian analysis of BHT massive gravity," JHEP 1101, 082 (2011) arXiv:1010.2596 [gr-qc]].

[9] M. Blagojevic and B. Cvetkovic, "Extra gauge symmetries in BHT gravity," JHEP 1103, 139 (2011) arXiv:1103.2388 [gr-qc]]. arXiv:0912.2944 [hep-th]].

[10] R. Banerjee, S. Gangopadhyay and D. Roy, "Hamiltonian analysis of symmetries in a massive theory of gravity," JHEP 1110 (2011) 121 arXiv:1108.4591 [gr-qc]].

[11] M. Henneaux, C. Teitelboim, "Quantization of gauge systems," Princeton, USA: Univ. Pr. (1992) $520 \mathrm{p}$.

[12] R. Utiyama, "Invariant theoretical interpretation of interaction," Phys. Rev. 1011597 (1956).

[13] T. W. B. Kibble, "Lorentz invariance and the gravitational field," J. Math. Phys. 2 212, (1961).

[14] D. W. Sciama, "On the analog between charge and spin in general relativity," p-415, Recent Developments in General Relativity, Festschrift for Leopold Infeld, Pergamon Press, New York (1962).

[15] F. W. Hehl, P. Von Der Heyde, G. D. Kerlick and J. M. Nester, "General Relativity With Spin And Torsion: Foundations And Prospects," Rev. Mod. Phys. 48, 393 (1976).

[16] M. Henneaux, C. Teitelboim, J. Zanelli, "Gauge Invariance And Degree Of Freedom Count," Nucl. Phys. B332, 169-188 (1990).

[17] R. Banerjee, H. J. Rothe and K. D. Rothe, "Hamiltonian approach to Lagrangian gauge symmetries," Phys. Lett. B 463, 248 (1999) arXiv:hep-th/9906072.

[18] R. Banerjee, H. J. Rothe and K. D. Rothe, "Master equation for Lagrangian gauge symmetries," Phys. Lett. B 479, 429 (2000) arXiv:hep-th/9907217].

[19] E. W. Mielke and P. Baekler, "Topological Gauge Model Of Gravity With Torsion," Phys. Lett. A 156, 399 (1991).

[20] P. Baekler, E. W. Mielke and F. W. Hehl, "Dynamical Symmetries In Topological 3-D Gravity With Torsion," Nuovo Cim. B 107, 91 (1992).

[21] P.A.M. Dirac, "Lectures on Quantum Mechanics," Dover Publications, New York, $200196 p$. 
[22] L. Castellani, "Symmetries In Constrained Hamiltonian Systems," Annals Phys. 143, 357 (1982).

[23] R. Banerjee, D. Roy, S. Samanta, "Lagrangian generators of the Poincare gauge symmetries," Phys. Rev. D82, 044012 (2010). [arXiv:1005.2904 [gr-qc]].

[24] S. Alexandrov, "The effective action and quantum gauge transformations," Phys. Rev. D 59, 125016 (1999) arXiv:hep-th/9807159]. 